\title{
Surface Density Function statistics in Hydrogen-air flames for different turbulent premixed combustion regimes
}

Nilanjan Chakraborty ${ }^{1 *}$, Markus Klein ${ }^{2}$, Dana Alwazzan ${ }^{1}$, Hong G. Im $^{3}$

${ }^{1}$ School of Engineering

University of Newcastle

Claremont Road, Newcastle

NE1 7RU, UK

Email: nilanjan.chakraboty@ncl.ac.uk; d.s.a.a.alwazzan@newcastle.ac.uk

${ }^{2}$ Universität der Bundeswehr München, Fakultät für Luft- und Raumfahrttechnik, LRT1, Werner-Heisenberg-Weg 39, 85577 Neubiberg, Germany

Email: markus.klein@unibw.de

${ }^{3}$ Clean Combustion Research Center, King Abdullah University of Science and Technology (KAUST), Thuwal 23955-6900, Saudi Arabia

Email: hong.im@kaust.edu.sa

* Corresponding author 


\begin{abstract}
The choice of reaction progress variable $c$ on the statistical behaviour of the surface density function (SDF) and the strain rates, which govern the evolution of SDF, have been analysed using a detailed chemistry Direct Numerical Simulation (DNS) database of freely propagating statistically planar $\mathrm{H}_{2}$-air flames with an equivalence ratio of 0.7 . The DNS database consists of three cases spanning the corrugated flamelets, thin reaction zones and broken reaction zones regimes of premixed turbulent combustion. For this analysis, the reaction progress variable is defined based on the mass fractions of $\mathrm{H}_{2}, \mathrm{O}_{2}$ and $\mathrm{H}_{2} \mathrm{O}$. The mean variations of the SDF and the flame displacement speed $S_{d}$ have also been found to be dependent on the choice of the reaction progress variable. The progressive weakening (strengthening) of the preferential alignment of the reaction progress variable gradient with the most extensive (compressive) principal strain rate with increasing Karlovitz number leads to changes in the behaviours of normal and tangential strain rates from one combustion regime to another. The differences in displacement speed statistics for different choices of reaction progress variable affect the behaviours of the normal strain rate due to flame propagation and curvature stretch. The relative thickening/thinning of the reaction layers of the major species has been explained in terms of the statistics of effective normal strain rate experienced by the $c$-isosurfaces for different choices of reaction progress variable.
\end{abstract}

Keywords: Premixed flame, Surface Density Function, normal strain rate, tangential strain rate, stretch rate 


\section{INTRODUCTION}

The modelling of turbulent premixed combustion and especially the closures of generalised Flame Surface Density (FSD) (Boger et al., 1998), $\Sigma_{g e n}=\overline{|\nabla c|}$, and scalar dissipation rate (SDR) (Chakraborty et al., 2011), $N_{c}=D \nabla c . \nabla c$, depend on the statistics of the Surface Density Function (SDF) (Kollmann and Chen, 1998), $|\nabla c|$, where $c$ is the reaction progress variable (RPV), $D$ is the progress variable diffusivity and the overbar indicates Reynolds averaging or Large Eddy Simulation filtering operation, as appropriate. The evolution of the SDF and its strain rate and curvature dependence have been analysed by a number of researchers (Pope, 1988; Candel and Poinsot, 1990; Chakraborty and Cant, 2005; Kim and Pitsch, 2007; Sankaran et al., 2007; Chakraborty and Klein, 2008, 2009; Chakraborty et al., 2008). Furthermore, the implications of $\nabla c$ alignment with local principal strain rates on the FSD and SDR transports have also been studied (Chakraborty and Swaminthan, 2007; Kim and Pitsch, 2007; Chakraborty et al., 2009; Hartung et al., 2008). A number of studies (Cifuentes et al., 2014; Cifuentes et al., 2015; Dopazo et al., 2015a,b; Dopazo and Cifuentes, 2016; Dopazo et al., 2017) have recently demonstrated the influences of strain rates arising from flame propagation on the SDF evolution based on simple chemistry Direct Numerical Simulation (DNS) data representing the flamelet regime of combustion. On the other hand, Wang et al. (2017) reported some important qualitative differences in high Karlovitz number flames in comparison to the findings reported in Cifuentes et al. (2014), Cifuentes et al. (2015) Dopazo et al. (2015a,b) and Dopazo and Cifuentes (2016).

To date, there has not been any analysis which compared the statistics of the SDF and the strain rates relevant to the SDF evolution for different regimes of turbulent premixed combustion. Moreover, in the context of detailed chemistry, the RPV cannot be defined uniquely and different definitions of $c$ based on different major species may lead to different SDF statistics. 
As such, the SDF statistics are expected to reflect the responses of different species reaction layers to the strain rate, depending on the definition of $c$. With the advances in computing power, researchers started to consider multiple species and solve individual scalar transport equations in the context of RANS (Fiorina et al., 2005) and even LES (Li and Kong, 2008; Vermoral et al., 2009). Hence it is essential to understand the sensitivity of the SDF statistics on the definition of RPV in order to ensure appropriate modelling of FSD and SDR transport. To address these issues in detail, the main objectives of this paper are:

(a) to demonstrate the differences in the statistical behaviours of the SDF and the strain rates which affect the SDF evolution for different combustion regimes and different definitions of the RPV;

(b) to provide physical explanations for the observed behaviours.

The rest of the paper will be organised as follows. The pertinent details about the mathematical background and numerical implementation are provided next section before the results are presented and discussed. The main findings will be summarised and conclusions are drawn at the end of this paper.

\section{MATHEMATICAL BACKGROUND \& NUMERICAL IMPLEMENTATION}

The RPV can be defined as: $c=\left(Y_{0}-Y\right) /\left(Y_{0}-Y_{\infty}\right)$ where $Y$ stands for the mass fraction of a chosen species, and the subscripts 0 and $\infty$ indicate values in the unburned and fully burned gases, respectively. Often the non-dimensional temperature $c_{T}=\left(\widehat{T}-T_{0}\right) /\left(T_{a d}-T_{0}\right)$ (where $\widehat{T}, T_{0}$ and $T_{a d}$ are the dimensional, unburned gas and adiabatic flame temperatures respectively) is also considered as the RPV but local differential diffusion effects may lead to super-adiabatic temperature (i.e. $c_{T}>1$ ) in $H_{2}$-air flames and thus invalidates its use as the RPV. Accordingly, $\left|\nabla c_{T}\right|$ may not provide a suitable measure of flame thickness in turbulent $H_{2}$-air flames and thus the SDF statistics based on $c_{T}$ have not been considered here. 
The evolution of the magnitude of the normal distance $\Delta x$ of two adjacent $c$-isosurfaces is given by (Cifuentes et al., 2014; Cifuentes et al., 2015; Dopazo et al., 2015a,b; Dopazo and Cifuentes, 2016; Dopazo et al., 2017):

$$
(\Delta x)^{-1}(d \Delta x / d t)=\left[N_{i} N_{j}\left(\partial u_{i} / \partial x_{j}\right)+N_{j} \partial S_{d} / \partial x_{j}\right]=a_{N}^{e f f}
$$

where $u_{i}$ and $N_{i}=-\left(\partial c / \partial x_{i}\right) /|\nabla c|$ are the $\mathrm{i}^{\text {th }}$ components of fluid velocity and flame normal, respectively, $d(\ldots) / d t=\partial(\ldots) / \partial t+V_{j}^{c} \partial(\ldots) / \partial x_{j}$ is the total derivative with respect to a reference frame attached with the flame with $V_{j}^{c}=\left(u_{j}+S_{d} N_{j}\right)$ being the $\mathrm{j}^{\text {th }}$ component of propagation velocity of a given $c$ isosurface. In eq. $1, a_{N}=N_{i} N_{j} \partial u_{i} / \partial x_{j}$ is the flame normal strain rate, $S_{d}=[\dot{w}+\nabla \cdot(\rho D \nabla c)] / \rho|\nabla c|$ is the displacement speed of a chosen $c$ iso-surface, where $\rho$ is the gas density, $\dot{w}=-\dot{w}_{Y} /\left(Y_{0}-Y_{\infty}\right)$ is the normalised reaction rate of species $Y$. Moreover, $a_{N}^{e f f}=a_{N}+N_{j} \partial S_{d} / \partial x_{j}$ is the effective normal strain rate which influences the evolution of $|\nabla c|$ in the following manner (Chakraborty and Cant, 2005; Kim and Pitsch, 2007; Sankaran et al., 2007; Chakraborty and Klein, 2008,2009; Chakraborty et al., 2008):

$$
\partial|\nabla c| / \partial t+\partial\left(u_{j}|\nabla c|\right) / \partial x_{j}=a_{T}|\nabla c|-\partial\left(S_{d} N_{j}|\nabla c|\right) / \partial x_{j}+2 S_{d} \kappa_{m}|\nabla c|
$$

where $\kappa_{m}=0.5\left(\partial N_{i} / \partial x_{i}\right)$ is the arithmetic mean of two principal flame curvatures and $a_{T}=$ $\left(\delta_{i j}-N_{i} N_{j}\right)\left(\partial u_{i} / \partial x_{j}\right)$ is the tangential strain rate. Using chain rules on both left and right hand sides of eq. 2 yields:

$$
\partial|\nabla c| / \partial t+V_{j}^{c} \partial|\nabla c| / \partial x_{j}=-a_{N}|\nabla c|-N_{j} \partial S_{d} / \partial x_{j}|\nabla c| \text { or }|\nabla c|^{-1}(d|\nabla c| / d t)=-a_{N}^{e f f}
$$

A comparison between eqs. 1 and 3 reveals that an increase in $a_{N}^{\text {eff }}$ increases the normal distance between $c$-iso-surfaces which leads to a decrease in $|\nabla c|$. In this context, it is also worthwhile to consider the evolution of the flame surface area $A$ (Pope, 1988; Candel and 
Poinsot, 1990; Cifuentes et al., 2014; Cifuentes et al., 2015; Dopazo et al., 2015a,b; Dopazo and Cifuentes, 2016; Dopazo et al., 2017):

$$
A^{-1}(d A / d t)=\left(a_{T}+2 S_{d} \kappa_{m}\right)=\left[\left(\delta_{i j}-N_{i} N_{j}\right)\left(\partial u_{i} / \partial x_{j}\right)+2 S_{d} \kappa_{m}\right]=a_{T}^{e f f}
$$

In eq. $4,2 S_{d} \kappa_{m}$ is an additional contribution to the tangential strain rate due to flame propagation and $a_{T}^{e f f}$ is the effective tangential strain rate (Cifuentes et al., 2014; Cifuentes et al., 2015; Dopazo et al., 2015a,b; Dopazo and Cifuentes, 2016; Dopazo et al., 2017). The quantities $a_{T}^{e f f}$ and $2 S_{d} \kappa_{m}$ are alternatively referred to as stretch rate and curvature stretch, respectively (Pope, 1988; Candel and Poinsot, 1990).

The statistics of $|\nabla c|$ and its evolution have been analysed here in terms of the statistical behaviours of $a_{N}, a_{T}$ and $S_{d}$ for RPVs based on $\mathrm{H}_{2}, \mathrm{O}_{2}$ and $\mathrm{H}_{2} \mathrm{O}$ mass fractions for freely propagating statistically planar atmospheric turbulent premixed $\mathrm{H}_{2}$-air flames with an equivalence ratio $\phi$ of 0.7 . This choice of $\phi$ is motivated by the fact that $\mathrm{H}_{2}$-air premixed flames remain thermo-diffusively neutral in that the flame speed is insensitive to stretch (Chen and Im, 2002). A three-dimensional DNS (Arias et al., 2016; Wacks et al., 2016) database, employing a detailed chemical mechanism with 9 steps and 19 chemical reactions (Burke et $a l ., 2012$ ), is considered here. The cases investigated here are representative of three regimes of combustion: case A: corrugated flamelets $(\mathrm{CF})(K a<1)$, case B: thin reaction zones (TRZ) $(1<K a<100)$ and case $\mathrm{C}$ : broken reaction zones (BRZ) regime $(K a>100)($ Peters, 2000). The numerical implementation has been discussed elsewhere (Arias et al., 2016; Wacks et al., 2016) and thus will not be repeated here. Turbulent inflow and outflow boundaries are taken in the direction of mean flame propagation and transverse boundaries are periodic. The inflow values of normalised root-mean-square turbulent velocity fluctuation $u^{\prime} / S_{L}$, turbulent length scale to flame thickness ratio $l_{T} / \delta_{t h}$, Damköhler number $D a=l_{T} S_{L} / u^{\prime} \delta_{t h}$, Karlovitz number 
$K a=\left(\rho_{0} S_{L} \delta_{t h} / \mu_{0}\right)^{0.5}\left(u^{\prime} / S_{L}\right)^{1.5}\left(l_{T} / \delta_{t h}\right)^{-0.5}$ and turbulent Reynolds number $R e_{t}=\rho_{0} u^{\prime} l_{T} /$ $\mu_{0}$ for all cases are presented in Table 1 where $\rho_{0}$ is the unburned gas density, $\mu_{0}$ is the unburned gas viscosity, $\delta_{t h}=\left(T_{a d}-T_{0}\right) / \max |\nabla T|_{L}$ is the thermal flame thickness and the subscript $L$ refers to unstrained laminar flame quantities.

The domain size is $20 \mathrm{~mm} \times 10 \mathrm{~mm} \times 10 \mathrm{~mm}(8 \mathrm{~mm} \times 2 \mathrm{~mm} \times 2 \mathrm{~mm})$ in cases A and B (case C), which is discretised by a uniform Cartesian grid of $512 \times 256 \times 256(1280 \times 320 \times 320)$ ensuring 10 grid points across $\delta_{t h}$. Simulations have been carried out until a quasi-steady state in terms of flame surface area is obtained (Arias et al., 2016; Wacks et al., 2016), and the simulation time (i.e. $1.0 l_{T} / u^{\prime}, 6.8 l_{T} / u^{\prime}$ and $6.7 l_{T} / u^{\prime}$ for cases A-C respectively) remains comparable to several previous analyses (Boger et al., 1994; Cifuentes et al., 2014; Charlette et al., 2002; Han and Huh, 2008; Reddy et al., 2012).

\section{RESULTS \& DISCUSSION}

The distributions of $c$ based on $\mathrm{H}_{2}$ and $\mathrm{H}_{2} \mathrm{O}$ mass fractions for cases A-C in $x_{1}-x_{3}$ mid-plane are shown in Fig. 1. The isocontours of $c$ remain parallel to each other in case A as energetic turbulent eddies do not penetrate into the flame. In contrast, eddies penetrate into the flame in cases $\mathrm{B}$ and $\mathrm{C}$ but the reaction zone in case $\mathrm{B}$ retains its quasi-laminar structure. In case $\mathrm{C}$, turbulent eddies penetrate into both preheat and reaction zones and cause considerable flame distortion. Figure 1 shows that $\mathrm{H}_{2}$ and $\mathrm{H}_{2} \mathrm{O}$ based RPVs are different especially on the burned gas side in cases B and $\mathrm{C}$.

The effect of turbulence on the flame thickness remains a controversial issue. The thickening of reaction layer in a mean sense under turbulent environment has been reported in previous experimental (O’Young and Bilger, 1997; Chen and Monsour, 1998; Chen and Bilger, 2002) 
and DNS (Sankaran et al., 2007; Wang et al., 2017) analyses, while contradictory evidences are also found in DNS (Hawkes and Chen, 2004) and experimental (Soika et al., 1998) studies. In this context, the flame thickness is commonly determined by the statistical average gradient in the RPV. To further examine this issue, the profiles of the mean values of $|\nabla c| \times \delta_{L}$ conditional on $c$ for cases A-C are shown in Figs. 2a-c for RPVs based on $\mathrm{H}_{2}, \mathrm{O}_{2}$ and $\mathrm{H}_{2} \mathrm{O}$ mass fractions respectively. Here, $\delta_{L}=1 / \max |\nabla c|_{L}$ is the reference flame thickness based on a laminar unstretched flame solution. As such, the peak mean value of $|\nabla c| \times \delta_{L}$ indicates a propensity towards flame thinning $(>1)$ or thickening $(<1)$ in a mean sense relative to the reference laminar flame condition.

Figure 2 shows that the flame thickening versus thinning depends on the choice of the RPVs. For RPVs based on $\mathrm{O}_{2}$ and $\mathrm{H}_{2} \mathrm{O}$, the peak mean value of $|\nabla c| \times \delta_{L}$ increases (thinner flame) from case A to case C. On the other hand, for $\mathrm{H}_{2}$-based RPV the flame thickens from case A to case $\mathrm{C}$, and even becomes slightly thicker than the laminar flame condition in case C. While a detailed analysis will follow, this behaviour is heuristically attributed to the fact that the $\mathrm{H}_{2}$ based flame thickness is larger due to the higher diffusivity, reaching out to farther upstream, thus being subjected to a stronger level of turbulence. This finding does not settle the open question because it is limited to the hydrogen-air flames for the flow conditions under study, but implies that a careful re-examination of the existing and new data is warranted by considering the sensitivity to the choice of the RPVs.

With this basic observation, Equations 2-4 provide a means to analyse the specific contributions from the statistical behaviours of $S_{d}, a_{N}$ and $a_{T}$ to the SDF, in order to explain why the reaction zone thicknesses for different species respond differently. 
First, as for the aerodynamic strain components, the mean values of flow dilatation $\nabla . \vec{u}$, normal strain rate $a_{N}$, and tangential strain rate $a_{T}$ conditional upon $c$ for different choices of RPV are shown in Figs. 3a-c, respectively. All quantities are normalized by the product with the flame time $\delta_{t h} / S_{L}$. The spatial distribution for all three quantities are seen to be distinct for the $\mathrm{H}_{2}$ based RPVs. The mean values of $\nabla \cdot \vec{u}$ remain at substantial values for cases $\mathrm{A}$ and $\mathrm{B}$, but are markedly smaller for case $\mathrm{C}$. The dilatation rate assumes mostly positive values in premixed flames due to heat release, but the effect is attenuated in case $\mathrm{C}$ as energetic eddies enter into the reaction zone and distribute the chemical reaction more widely across the flame thickness.

The mean value of $a_{N}$ remains predominantly positive in cases $\mathrm{A}$ and $\mathrm{B}$, whereas it is negative throughout the flame for case C. The normal strain rate $a_{N}$ can be expressed as $a_{N}=$ $\left(e_{\alpha} \cos ^{2} \alpha+e_{\beta} \cos ^{2} \beta+e_{\gamma} \cos ^{2} \gamma\right)$ where $e_{\alpha}, e_{\beta}$ and $e_{\gamma}$ are the most extensive, intermediate and most compressive principal strain rates respectively and $\alpha, \beta$ and $\gamma$ are the angles between $\nabla c$ and the eigenvectors associated with $e_{\alpha}, e_{\beta}$ and $e_{\gamma}$, respectively. It has been shown (Chakraborty and Swaminathan, 2007; Kim and Pitsch, 2007; Chakraborty et al., 2009; Hartung et al., 2008) that $\nabla c$ aligns strongly with $e_{\alpha}$ when the strain rate due to flame normal acceleration dominates over turbulent straining and this is highly probable for flames with $D a>1$ (Chakraborty and Swaminathan, 2007; Chakraborty et al., 2009). In contrast, $\nabla c$ preferentially aligns with $e_{\gamma}$ when turbulent strain rate overcomes the strain rate arising from flame normal acceleration, which is highly probable for $D a<1$ (Chakraborty and Swaminathan, 2007; Chakraborty et al., 2009). Accordingly, $\nabla c$ preferentially aligns with $e_{\alpha}$ for cases $\mathrm{A}$ and $\mathrm{B}$, and thus yielding a mean positive value of $a_{N}$, while $\nabla c$ aligns with $e_{\gamma}$ in case $\mathrm{C}$ to give rise to negative mean values of $a_{N}$. Referring to eq. 3 , this implies that the 
normal flow strain has a net flame thickening effect for cases $\mathrm{A}$ and $\mathrm{B}$, and thinning effect for case C. This qualitative behaviour is independent of the choice of the RPV species.

Regarding the effect on the flame surface, the mean value of $a_{T}=\nabla \cdot \vec{u}-a_{N}$ is determined by the relative magnitudes and signs of $\nabla \cdot \vec{u}$ and $a_{N}$. In case A, the mean values of $\nabla \cdot \vec{u}$ and $a_{N}$ remain close to each other to yield a small positive mean value of $a_{T}$. The extent of the alignment of $\nabla c$ with $e_{\alpha}$ is relatively weaker in case B than in case A and that leads to smaller positive mean value of $a_{N}$ in case $\mathrm{B}$ in comparison to that in case A. With $\nabla \cdot \vec{u}$ being comparable for cases $\mathrm{A}$ and $\mathrm{B}$, this results in a greater value of $a_{T}=\nabla \cdot \vec{u}-a_{N}$ in case $\mathrm{B}$ than in case A. For case $\mathrm{C}$, the large negative mean value $a_{N}$ eclipses the reduced mean positive value of $\nabla \cdot \vec{u}$ to lead to a large positive mean value of $a_{T}=\nabla \cdot \vec{u}-a_{N}$. Referring to eq. 4 , this implies that the aerodynamic tangential strain rate continues to increase from case $\mathrm{A}$ to $\mathrm{C}$, leading to an increased flame surface area.

Next, the effects associated with the flame propagation are examined. The variations of mean values of $S_{d} / S_{L}$ conditional upon $c$ for cases A-C are shown in Figs. 4 a-c for $\mathrm{H}_{2}, \mathrm{O}_{2}$ and $\mathrm{H}_{2} \mathrm{O}$ based RPVs respectively. All $S_{d}$ related quantities have been shown for $0.05 \leq c \leq 0.95$ in Fig. 4 and subsequent figures to avoid singularities in the displacement speed evaluation. Figure 4 shows that $S_{d} / S_{L}$ for $\mathrm{H}_{2}$ based RPV increases from unburned to burned gas side before reaching a peak value close to $c \approx 1.0$. For $\mathrm{O}_{2}$ and $\mathrm{H}_{2} \mathrm{O}$ based RPVs, the peak mean value of $S_{d} / S_{L}$ is obtained close to the middle of the flame-front but slightly skewed towards the burned gas side and this suggests $\rho S_{d} \neq \rho_{0} S_{L}$ for these cases as $\rho$ monotonically decreases with increasing $c$. 
The differences in the mean behaviours of $S_{d} / S_{L}$ for $\mathrm{H}_{2}, \mathrm{O}_{2}$ and $\mathrm{H}_{2} \mathrm{O}$ can be explained by examining the statistical behaviours of $S_{d}=S_{r}+S_{n}+S_{t}$ where $S_{r}=\dot{w} / \rho|\nabla c| ; S_{n}=$ $\vec{N} . \nabla(\rho D \vec{N} \cdot \nabla c) / \rho|\nabla c|$ and $S_{t}=-2 D \kappa_{m}$, which are the reaction, normal diffusion and tangential diffusion components of $S_{d}$, respectively (Peters et al., 1998; Echekki and Chen, 1999; Im and Chen, 2002). The variations of mean values of $S_{r} / S_{L}, S_{n} / S_{L}$ and $S_{t} / S_{L}$ conditional upon $c$ are shown in Fig. 5 which reveals that the mean values of $\left(S_{r}+S_{n}\right) / S_{L}$ (not shown) and $S_{d} / S_{L}$ remain close to each other because the mean contribution of $S_{t}=-2 D \kappa_{m}$ is negligible in statistically planar flames. Figure 5 shows that the mean value of $S_{r} / S_{L}$ remains positive throughout the flame-front, whereas the mean value of $S_{n} / S_{L}$ assumes positive (negative) values towards the unburned (burned) gas side of the flame-front. However, the qualitative distributions of $S_{r} / S_{L}$ and $S_{n} / S_{L}$ are different for each species, which contributes to the differences in of $S_{d} / S_{L}$ for different RPVs.

To assess contributions from the flame propagation to the SDF as appearing in eq. 3 , the mean values of $N_{j} \partial S_{d} / \partial x_{j}, N_{j} \partial\left(S_{r}+S_{n}\right) / \partial x_{j}$ and $N_{j} \partial S_{t} / \partial x_{j}$, normalised with $\delta_{t h} / S_{L}$, conditional upon $c$ for different choices of RPV for cases A-C are shown in Figs. 6a-c respectively. For cases $\mathrm{A}$ and $\mathrm{B}$, it is evident that the behaviour of $N_{J} \partial S_{d} / \partial x_{j}$ is dominated by $N_{J} \partial\left(S_{r}+\right.$ $\left.S_{n}\right) / \partial x_{j}$, while the effect of $N_{J} \partial S_{t} / \partial x_{j}$ is minimal. Although not shown here, the mean values of $N_{J} \partial S_{r} / \partial x_{j}$ and $N_{J} \partial S_{n} / \partial x_{j}$ are close to each other at large magnitudes but opposite in sign, leading to the undulations in the net values. The average value across the entire region for $c$ is negative as a net, and contributes to the overall flame thinning, as seen in Fig. 2.

In case $\mathrm{C}$, however, the magnitudes of $N_{J} \partial S_{t} / \partial x_{j}$ and $N_{J} \partial\left(S_{r}+S_{n}\right) / \partial x_{j}$ remain comparable, with magnitudes much larger than in cases $\mathrm{A}$ and $\mathrm{B}$. The positive mean values of $N_{J} \partial S_{t} / \partial x_{j}$ 
dominate over negative mean values of $N_{J} \partial\left(S_{r}+S_{n}\right) / \partial x_{j}$ to yield mean positive values of $N_{J} \partial S_{d} / \partial x_{j}$, leading to the flame thickening. Incidentally, as the $c$-isosurfaces are not parallel to each other in cases B and C, especially on the burned gas side (see Fig. 1), the spatial variation of $\kappa_{m}$ along with high $D$ leads to large magnitudes of $N_{J} \partial S_{t} / \partial x_{j}=$ $-2 N_{J} \partial\left(D \kappa_{m}\right) / \partial x_{j}$ towards the burned gas side in these cases. According to scaling arguments by Peters (2000), the contribution of $S_{t}$ to $S_{d}$ strengthens with increasing $K a$ and thus the contribution of $N_{J} \partial S_{t} / \partial x_{j}$ is likely to play a dominant role for $K a \gg 1$ flames, consistent with the present observation in case $\mathrm{C}$.

Figures $6 \mathrm{~d}-\mathrm{f}$ further shows the flame propagation effect coupled with the curvature stretch $2 S_{d} \kappa_{m}$, i.e. the last term in eq. 2 , which assumes predominantly negative values for all cases except some positive values towards the burned gas side. The mean contribution of $2\left(S_{r}+\right.$ $\left.S_{n}\right) \kappa_{m}$ remains positive for all RPVs considered here. However, the deterministically negative mean contribution of $2 S_{t} \kappa_{m}$ dominates over $2\left(S_{r}+S_{n}\right) \kappa_{m}$ to result in the mostly negative mean values of $2 S_{d} \kappa_{m}$. The aforementioned strong $\kappa_{m}$ variation and high values of $D$ are responsible for large magnitudes of $2 S_{t} \kappa_{m}=-4 D \kappa_{m}^{2}$ towards the burned gas side of the flame-front. The differences in $S_{d}$ statistics and $c$ distributions contribute to the qualitative and quantitative differences in the mean behaviours of $N_{J} \partial S_{d} / \partial x_{j}$ and $2 S_{d} \kappa_{m}$ for different RPV definitions.

The mean values of $a_{N}^{e f f}$ conditional upon $c$ for different choices of RPV for cases A-C are shown in Fig. 7a-c. According to eq. 3, the effective normal strain rate assumes both positive and negative values within the flame-front for cases A and B, whereas the mean value of $a_{N}^{\text {eff }}$ remains positive throughout the flame-front for case C. A comparison between Figs. 3 and 6 reveals that the undulations in mean values of $a_{N}^{\text {eff }}$ originate due to the undulations of the mean 
values of $N_{j} \partial S_{d} / \partial x_{j}$. The mean value of the resulting evolution of the SDF $|\nabla c|^{-1}(\partial|\nabla c| / \partial t)$ is also shown in Figs. 7a-c for all choices of $c$. A comparison between Figs. 2a-c and 7a-c reveals that mean $|\nabla c|^{-1}(\partial|\nabla c| / \partial t) \times \delta_{t h} / S_{L}$ assumes positive values at the location of the peak mean value of SDF for $\mathrm{O}_{2}$ and $\mathrm{H}_{2} \mathrm{O}$ based RPVs, which is consistent with flame thinning observed in these cases. By contrast, the mean $|\nabla c|^{-1} \partial|\nabla c| / \partial t$ assumes a positive value at the location of the peak mean value of $|\nabla c|$ for cases A and B for $\mathrm{H}_{2}$ based RPV, whereas a small negative value is obtained at the location of the peak mean value of $|\nabla c|$ in case $C$, which is also in accordance with the flame thinning (thickening) in cases A and B (case C) in Fig. 2.

Finally, Figs. 7d-f show the variation of the mean stretch $a_{T}^{e f f}$ conditional upon $c$ for different choices of RPV for cases A-C, which indicate that the mean $a_{T}^{\text {eff }}$ remains predominantly negative for case $\mathrm{C}$, whereas the profile of the mean $a_{T}^{\text {eff }}$ undulates between positive and negative values with a bias towards positive values for cases A and B. The small imbalance between comparable magnitudes of $a_{T}$ and $2 S_{d} \kappa_{m}$ is responsible for the undulations in the mean $a_{T}^{\text {eff }}$ profiles in cases A-C. A similar behaviour was also reported in a recent simple chemistry based DNS analysis (Dopazo et al., 2017). A positive (negative) value of $a_{T}^{e f f}$ is indicative of flame area generation (destruction). The volume-integrated heat release rate $\Omega=$ $\int_{V} \dot{\omega}_{\mathrm{T}} d V$ (where $\dot{\omega}_{\mathrm{T}}$ is the heat release rate) in turbulent flames normalised by its corresponding laminar value assumes a value of about 3.5 for cases $\mathrm{A}$ and $\mathrm{C}$ and 5.0 for case B. Although $\Omega$ may not have a one-to-one relation with the flame surface area evaluated by the volume-integral of $|\nabla c|$ based on a single RPV definition in the context of multi-species systems, high values of flame surface area are still associated with high values of $\Omega$. The greater probability of having positive values of $a_{T}^{\text {eff }}$ in case B than in case C yields a higher value of $\Omega$ in case B. Moreover, the greater likelihood of positive values of $a_{T}^{\text {eff }}$ in case A than in case 
C leads to comparable values of $\Omega$ in these two cases in spite of case A having much smaller $u^{\prime} / S_{L}$ than in case $\mathrm{C}$ (see Table 1 ). The high values of $u^{\prime} / S_{L}$ in case $\mathrm{C}$ tend to produce highly wrinkled $c$ isosurfaces, and a combination of Huygens propagation and flame-flame interaction tends to produce a flat flame surface and this is reflected in the negative mean value of $a_{T}^{\text {eff }}$.

\section{CONCLUSIONS}

The effects of the choice of RPV definition on the mean behaviours of the SDF (i.e. $|\nabla c|$ ) and the strain rates affecting $|\nabla c|$ transport have been analysed using a detailed chemistry DNS database of statistically planar $\mathrm{H}_{2}$-air premixed flames with an equivalence ratio of 0.7 spanning the $\mathrm{CF}, \mathrm{TRZ}$ and BRZ regimes of combustion. For each case the SDF and strain rate statistics have been analysed for RPVs defined based on $\mathrm{H}_{2}, \mathrm{O}_{2}$ and $\mathrm{H}_{2} \mathrm{O}$ mass fractions. The differences in $|\nabla c|$ statistics for different definitions of RPV have been explained in terms of the statistics of strain rates in the flame normal and tangential directions. The dilatation rate effects weaken in the BRZ regime, and the alignment of $\nabla c$ with the most extensive (compressive) principal strain rate weakens (strengthens) from the CF to the TRZ regime to the BRZ regime. This leads to differences in the mean behaviours of normal and tangential strain rates from one case to another. The mean behaviours of $S_{d}$ and its components for different choices of the RPV have been found to be qualitatively different. This leads to differences in the normal strain rate arising from flame propagation and the curvature stretch for different choices of RPV. The sensitivity of the statistics of SDF on the definition of RPV indicates that the sub-models of the FSD and SDR transports need to accurately capture the respective behaviours of the unclosed terms for different definitions of RPV in order to be applicable in a multi-species system.

\section{ACKNOWLEDGEMENTS}


Part of the work presented in this study was sponsored by competitive research funding from King Abdullah University of Science and Technology (KAUST). The work made use of computational resources at KAUST Supercomputing Laboratory and ARCHER at Engineering and Physical Sciences Research Council (EPSRC). 


\section{REFERENCES}

Arias P.G., Chaudhuri S., Uranakara H.A., Im H.G. 2016. Direct numerical simulations of statistically stationary turbulent premixed flame, Combust. Sci. Technol., 188, 1182.

Boger, M., Veynante, D., Boughanem, H., Trouvé, A. 1998. Direct Numerical Simulation analysis of flame surface density concept for Large Eddy Simulation of turbulent premixed combustion, Proc. Combust. Inst., 27, 917.

Burke M.P., Chaos M., Ju Y. Dryer F.L., Klippenstein S.J. 2012. Comprehensive H2-O2 kinetic model for high-pressure combustion. Int. J. Chem. Kin.,44, 444.

Candel, S.M., Poinsot, T.J. 1990. Flame stretch and the balance equation for the flame area, Combust. Sci. Technol., 70,1.

Chakraborty, N., Cant, R.S. 2005. Effects of strain rate and curvature on Surface Density Function transport in turbulent premixed flames in the thin reaction zones regime, Phys. Fluids, 17,65108 .

Chakraborty, N., Cant, R.S. 2005b. Influence of Lewis Number on curvature effects in turbulent premixed flame propagation in the thin reaction zones regime, Phys. Fluids, 17,105105 .

Chakraborty, N., Swaminathan, N. 2007. Influence of Damköhler number on turbulence-scalar interaction in premixed flames, Part I: Physical Insight, Phys. Fluids, 19, 045103.

Chakraborty, N., Klein, M. 2008. Influence of Lewis number on the Surface Density Function transport in the thin reaction zones regime for turbulent premixed flames, Phys. Fluids, 20, 065102.

Chakraborty, N., Hawkes, E.R., Chen, J.H., Cant, R.S. 2008. Effects of strain rate and curvature on Surface Density Function transport in turbulent premixed $\mathrm{CH}_{4}$-air and $\mathrm{H}_{2}$-air flames: A comparative study, Combust. Flame, 154,259. 
Chakraborty, N., Klein, M., Swaminathan, N. 2009. Effects of global flame curvature on the Surface Density Function transport in Turbulent premixed flame kernels in the Thin Reaction Zones regime. Proc. Combust. Inst., 321435.

Chakraborty, N., Klein, M., Swaminathan, N. 2009. Effects of Lewis number on reactive scalar gradient alignment with local strain rate in turbulent premixed flames, Proc. Combust. Inst., 32,1409 .

Chakraborty, N., Champion, M., Mura, A., Swaminathan, N. 2011. Scalar dissipation rate approach to reaction rate closure, Turbulent premixed flame, (Eds. Swaminathan, N., Bray, K.N.C.), Cambridge University Press, 1st Edition, Cambridge, UK, pp. 74-102, 2011.

Charlette, F., Meneveau, C., Veynante, D. 2002 A power law wrinkling model for LES of premixed turbulent combustion, Part I: Non dynamic formulation and initial tests, Combust. Flame, 131, 159.

Chen, Y.-C., Monsour, M. S. 1998. Investigation of flame broadening in turbulent premixed flames in the thin reaction-zones .Combust. Inst., 27, 811.

Chen, Y.-C., Bilger, R. W. 2002. Experimental investigation of three-dimensional flame-front structure in premixed turbulent combustion-I: Hydrocarbon/air Bunsen flames, Combust. Flame, 131, 400.

Cifuentes, L., Dopazo, C., Martin, J., Jimenez, C. 2014. Local flow topologies and scalar structures in a turbulent premixed flame, Phys. Fluids, 26, 065108.

Cifuentes, L., Dopazo, C., Martin, J., Domingo, P., Vervisch, L. 2015. Local volumetric dilatation rate and scalar geometries in a premixed methane-air turbulent jet flame, Proc. Comb. Inst., 35, 1295.

Dopazo, C., Cifuentes, L., Martin, J., Jimenez, C. 2015. Strain rates normal to approaching isoscalar surfaces in a turbulent premixed flame, Combust. Flame, 162, 1729. 
Dopazo, C., Cifuentes, L., Hierro, J., Martin, J. 2015. Micro-scale mixing in turbulent constant density reacting flows and premixed combustion, Flow Turb. Comb., 96, 547.

Dopazo, C., Cifuentes, L. 2016. The physics of scalar gradients in turbulent premixed combustion and its relevance to modeling, Combust. Sci. Technol., 188, 1376.

Dopazo, C., Cifuentes, L., Alwazzan, D., Chakraborty, N. 2017. Influence of the Lewis number on effective strain rates in weakly turbulent premixed combustion, Combust. Sci. Technol., DOI: $10.1080 / 00102202.2017 .1398744$.

Echekki, T., and Chen, J.H. 1999. Analysis of the Contribution of Curvature to Premixed Flame Propagation, Combust. Flame, 118, 303.

Fiorina, B., Gicquel, O., Vervisch, L., Darabiha, N. 2005. Premixed turbulent combustion modeling using tabulated detailed chemistry and PDF, Proc. Combust. Inst., 30,867.

Han, I., Huh, K. 2008. Roles of displacement speed on evolution of flame surface density for different turbulent intensities and Lewis numbers in turbulent premixed combustion, Combust. Flame, 152, 194-205.

Hartung, G., Hult, J., Kaminski, C.F., Rogerson, J.W., Swaminathan, N., 2008. Effect of heat release on turbulence and scalar-turbulence interaction in premixed combustion, Phys. Fluids, $20,035110$.

Hawkes, E. R., Chen, J. H. 2006. Comparison of direct numerical simulation of lean premixed methane-air flames with strained laminar flame calculations, Combust. Flame, 144, 112. Im, H.G., and Chen, J.H., 2002. Preferential diffusion effects on the burning rate of interacting turbulent premixed Hydrogen-Air flames, Combust. Flame, 126, 246.

Kim, S.H., Pitsch, H. 2007. Scalar gradient and small-scale structure in turbulent premixed combustion, Phys. Fluid 19,115104.

Kollmann, W., Chen, J.H. 1998. Pocket formation and the flame surface density equation, Proc. Combust. Inst., 27, 927. 
Li, S.C., Kong, Y.H. 2008. Diesel combustion modelling using les turbulence model with detailed chemistry. Combust. Theor. Model, 12, 205.

O’Young, F., Bilger, R. W. 1997. Scalar gradient and related quantities in turbulent premixed flames, Combust. Flame, 109, 683.

Peters, N., Turbulent Combustion, Cambridge Monograph on Mechanics, Cambridge University Press, Cambridge (2000).

Peters, N., Terhoeven, P., Chen, J.H., and Echekki, T. 1998. Statistics of Flame Displacement Speeds from Computations of 2-D Unsteady Methane-Air Flames, Proc. Combust. Inst., 27,833 .

Reddy, H., Abraham, J. 2012. Two-dimensional direct numerical simulation evaluation of the flame-surface density model for flames developing from an ignition kernel in lean methane/air mixtures under engine conditions, Phys. Fluids, 24,105108.

Sankaran, R., Hawkes, E.R., Chen, J.H., Lu, T., Law, C.K. 2007. Structure of a spatially developing turbulent lean methane-air Bunsen flame, Proc. Combust. Inst. 31, 1291.

Soika, A., Dinkelacker, F., Leipertz, A. 1998. Measurement of resolved flame structure with constant Reynolds number, Proc. Comb. Inst., 27,785.

Vermorel, O., Richard, S., Colin, O., Angelberger, C., Benkenida, A., Veynante D. 2005. Towards the understanding of cyclic variability in a spark ignited engine using multicycle les. Combust. Flame, 156, 1525.

Wacks, D.H., Chakraborty, N., Klein, M., Arias, P.G., Im, H.G. 2016. Flow topologies in different regimes of premixed turbulent combustion: A direct numerical simulation analysis, Phys. Rev. F, 1, 083401. 
Table 1

List of inflow turbulence parameters

\begin{tabular}{|c|c|c|c|c|c|}
\hline Case & $\boldsymbol{u}^{\prime} / \boldsymbol{S}_{\boldsymbol{L}}$ & $\boldsymbol{l}_{\boldsymbol{T}} / \boldsymbol{\delta}_{\boldsymbol{t h}}$ & $\boldsymbol{R e}_{\boldsymbol{t}}$ & $\mathrm{Da}$ & $\mathrm{Ka}$ \\
\hline $\mathrm{A}$ & 0.7 & 14.0 & 227 & 20.0 & 0.75 \\
\hline B & 5 & 14.0 & 1623 & 2.8 & 14.4 \\
\hline C & 14 & 4.0 & 1298 & 0.29 & 126 \\
\hline
\end{tabular}




\section{FIGURE CAPTIONS}

Fig. 1: Distributions of RPV based on $\mathrm{H}_{2}$ ( $1^{\text {st }}$ row) and $\mathrm{H}_{2} \mathrm{O}$ ( $2^{\text {nd }}$ row) mass fractions in the central $x_{1}-x_{3}$ mid-plane for cases A-C $\left(1^{\text {st }}-3^{\text {rd }}\right.$ columns). The $c=0.1-0.9$ (in steps of 0.1 ) isolines are shown by magenta line.

Fig. 2: Variations of mean values of $|\nabla c| \times \delta_{L}$ conditional on $c$ for cases $A-C$ for (a) $\mathrm{H}_{2}$, (b) $\mathrm{O}_{2}$ and (c) $\mathrm{H}_{2} \mathrm{O}$ based RPVs. Here and in the remaining figures $c$ refers to the RPV defined based on the respective species mass fraction considered.

Fig. 3: Variations of $\nabla \cdot \vec{u}$ (solid line), $a_{N}$ (line with circle) and $a_{T}$ (dotted line), normalized with $\delta_{t h} / S_{L}$, conditional upon $c$ for cases (a-c) A-C. The results for RPV definitions based on $\mathrm{H}_{2}, \mathrm{O}_{2}$ and $\mathrm{H}_{2} \mathrm{O}$ mass fractions are shown by purple, green and brown lines respectively in Figs. 3,6 and-7.

Fig. 4: Variations of mean values of $S_{d} / S_{L}$ conditional upon $c$ for cases A-C for (a) $\mathrm{H}_{2}$, (b) $\mathrm{O}_{2}$ and (c) $\mathrm{H}_{2} \mathrm{O}$ based RPVs.

Fig. 5: Variations of mean values of $S_{\alpha} / S_{L}$ (where $\alpha=\mathrm{r}$ (solid line), $\mathrm{n}$ (broken line) and $\mathrm{t}$ (line with circle)) conditional upon $c$ for cases A-C for (a) $\mathrm{H}_{2}$, (b) $\mathrm{O}_{2}$ and (c) $\mathrm{H}_{2} \mathrm{O}$ based RPVs. The same colour code as in Fig. 2 is used.

Fig. 6: Variations of (a-c) $N_{J} \partial S_{\alpha} / \partial x_{j}\left(1^{\text {st }}\right.$ column) and (d-f) $2 S_{\alpha} \kappa_{m}$ (2 $2^{\text {nd }}$ column), normalized with $\delta_{t h} / S_{L}$, for (where $\alpha=\mathrm{d}$ (solid line), $\mathrm{r}+\mathrm{n}$ (broken line) and $\mathrm{t}$ (line with circle)) conditional upon $c$ for cases A-C $\left(1^{\text {st }}-3^{\text {rd }}\right.$ row $)$.

Fig. 7: Variations of (a-c) $a_{N}^{e f f}$, (solid line) $|\nabla c|^{-1}(\partial|\nabla c| / \partial t)$ (line with o) (1 ${ }^{\text {st }}$ column) and (d-f) $a_{T}^{e f f}\left(2^{\text {nd }}\right.$ column), normalized with $\delta_{t h} / S_{L}$, conditional upon $c$ for cases A-C $\left(1^{\text {st }}-3^{\text {rd }}\right.$ row). 

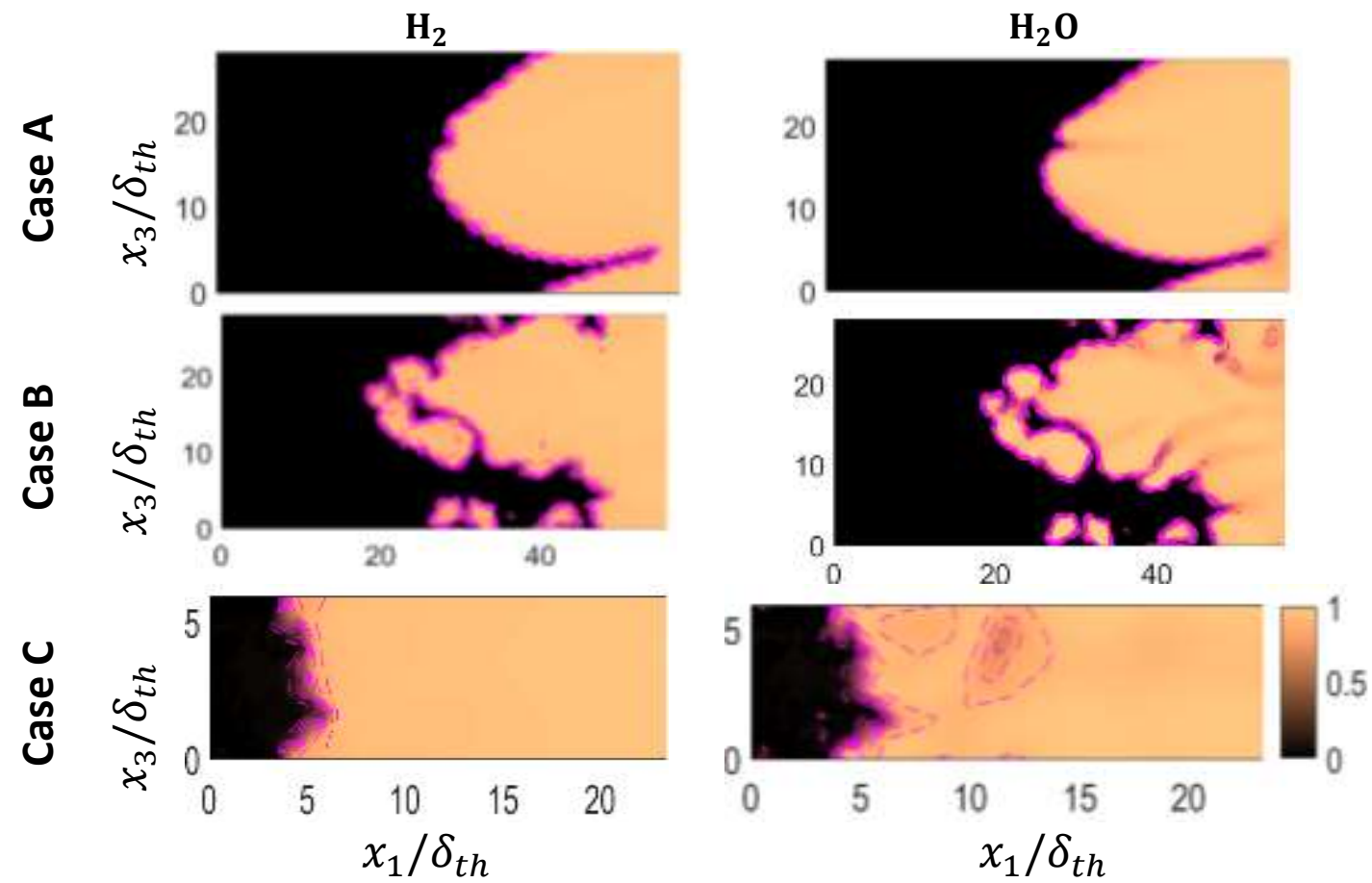

Fig. 1: Distributions of RPV based on $\mathrm{H}_{2}\left(1^{\text {st }}\right.$ column) and $\mathrm{H}_{2} \mathrm{O}$ ( $2^{\text {nd }}$ column) mass fractions in the central $x_{1}-x_{3}$ mid-plane for cases A-C $\left(1^{\text {st }}-3^{\text {rd }}\right.$ rows $)$. The $c=0.1-$ 0.9 (in steps of 0.1) isolines are shown by magenta lines. 

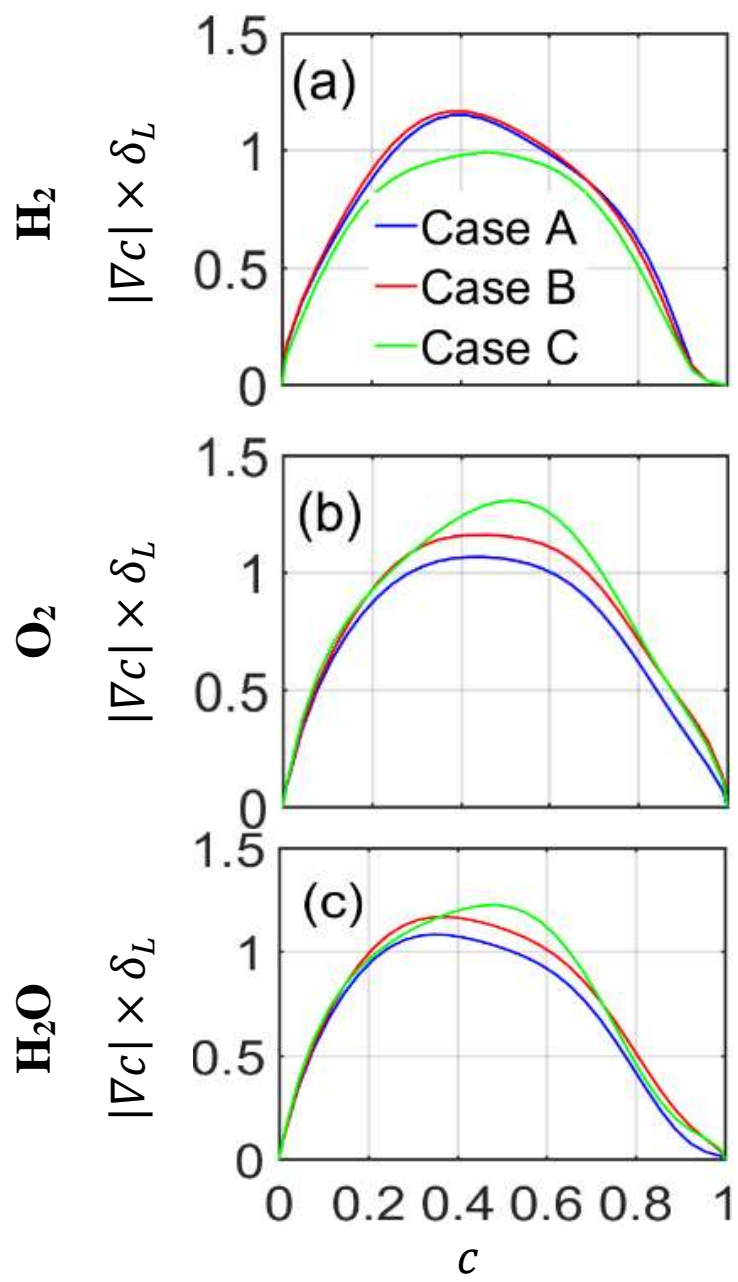

Fig. 2: Variations of mean values of $|\nabla c| \times \delta_{L}$ conditional on $c$ for cases A-C for (a) $\mathrm{H}_{2}$, (b) $\mathrm{O}_{2}$ and (c) $\mathrm{H}_{2} \mathrm{O}$ based RPVs. Here and in the remaining figures $c$ refers to the RPV defined based on the respective species mass fraction considered. 

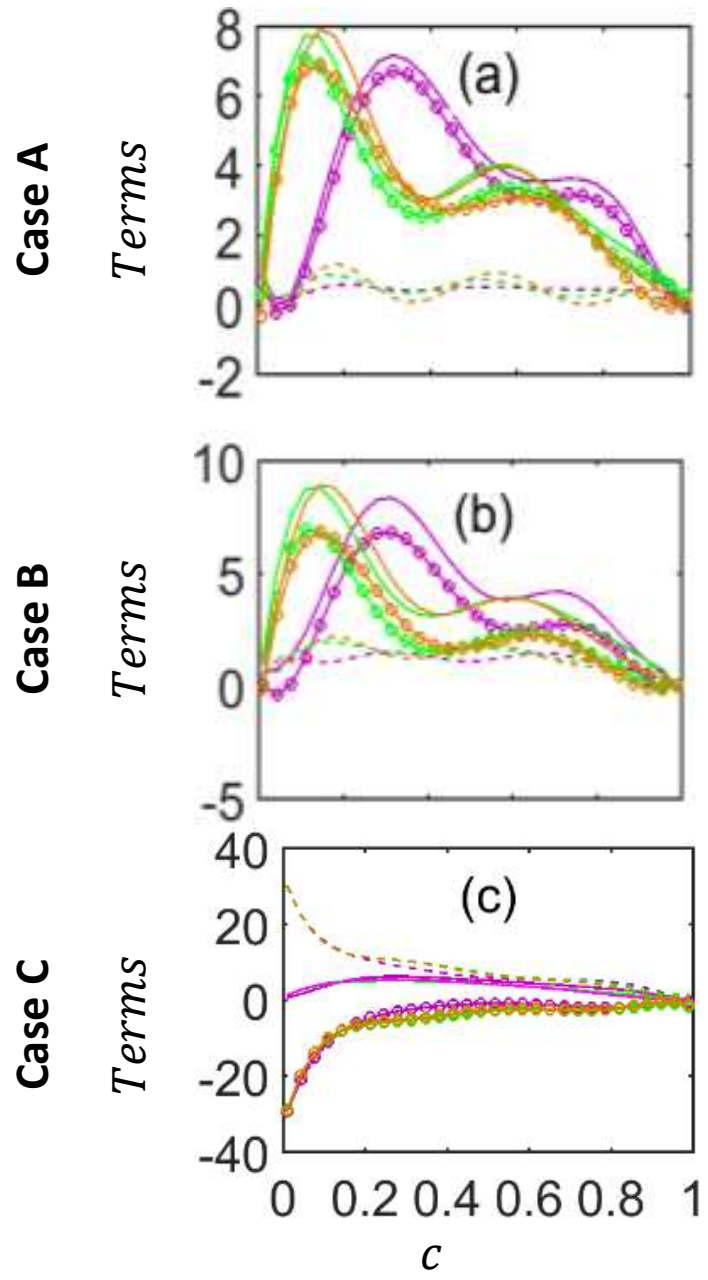

Fig. 3: Variations of $\nabla \cdot \vec{u}$ (solid line), $a_{N}$ (line with circle) and $a_{T}$ (dotted line), normalized with $\delta_{t h} / S_{L}$, conditional upon $c$ for cases (a-c) A-C. The results for RPV definitions based on $\mathrm{H}_{2}, \mathrm{O}_{2}$ and $\mathrm{H}_{2} \mathrm{O}$ mass fractions are shown by purple, green and brown lines respectively in Figs. 3,6 and-7. 


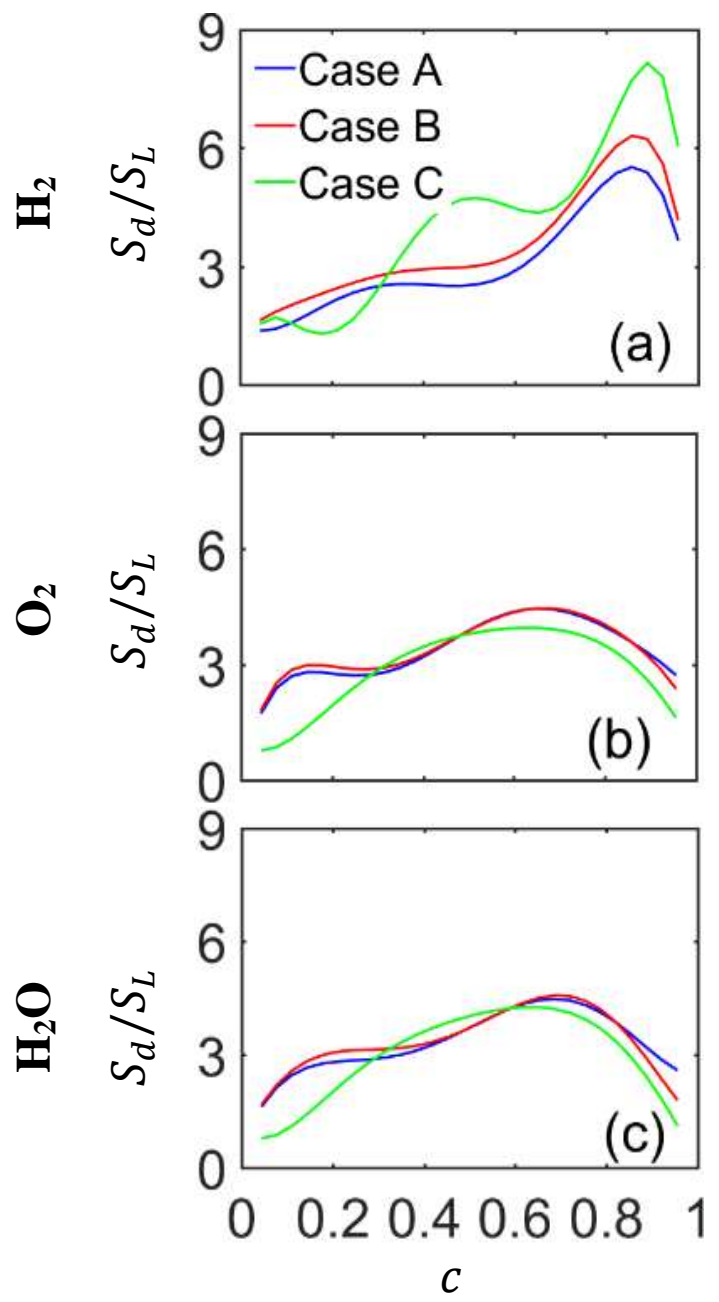

Fig. 4: Variations of mean values of $S_{d} / S_{L}$ conditional upon $c$ for cases A-C for (a) $\mathrm{H}_{2}$, (b) $\mathrm{O}_{2}$ and (c) $\mathrm{H}_{2} \mathrm{O}$ based RPVs. 


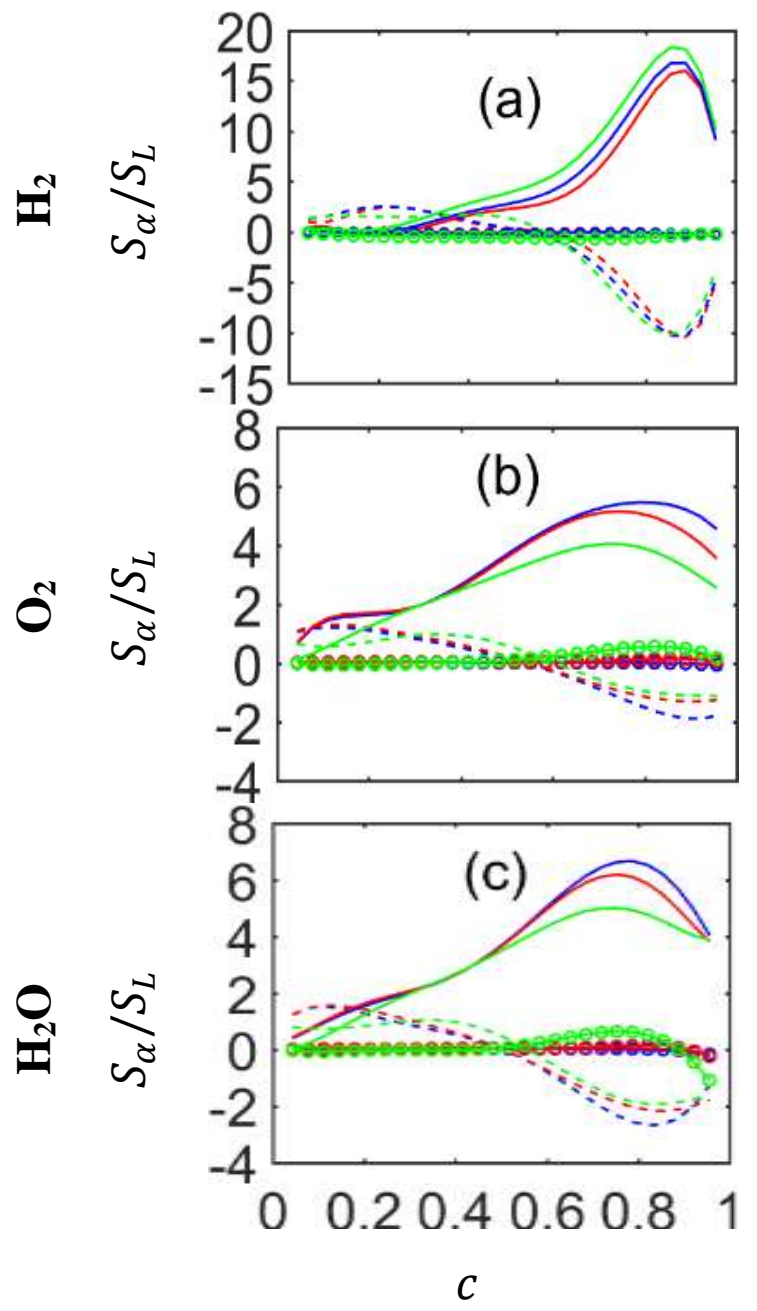

Fig. 5: Variations of mean values of $S_{\alpha} / S_{L}$ (where $\alpha=\mathbf{r}$ (solid line), n (broken line) and $t$ (line with circle)) conditional upon $c$ for cases A-C for (a) $\mathrm{H}_{2}$, (b) $\mathrm{O}_{2}$ and (c) $\mathrm{H}_{2} \mathrm{O}$ based RPVs. The same colour code as in Fig. 2 is used. 

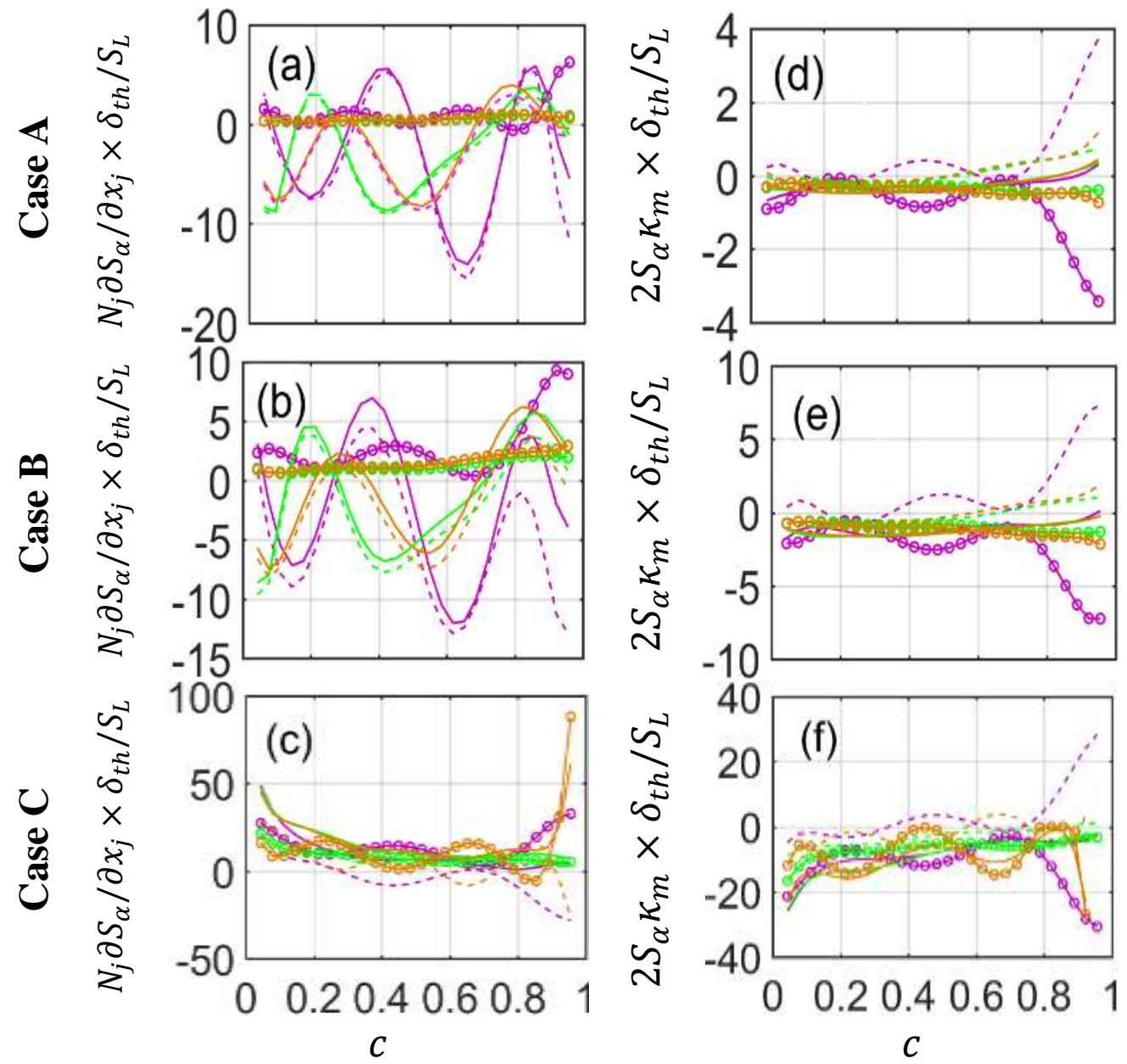

Fig. 6: Variations of (a-c) $N_{J} \partial S_{\alpha} / \partial x_{j}$ (1 $1^{\text {st }}$ column) and (d-f) $2 S_{\alpha} \kappa_{m}$ (2 ${ }^{\text {nd }}$ column), normalized with $\delta_{t h} / S_{L}$, for (where $\alpha=\mathrm{d}$ (solid line), r+n (broken line) and $\mathrm{t}$ (line with circle)) conditional upon $c$ for cases A-C $\left(1^{\text {st }}-3^{\text {rd }}\right.$ row $)$. 

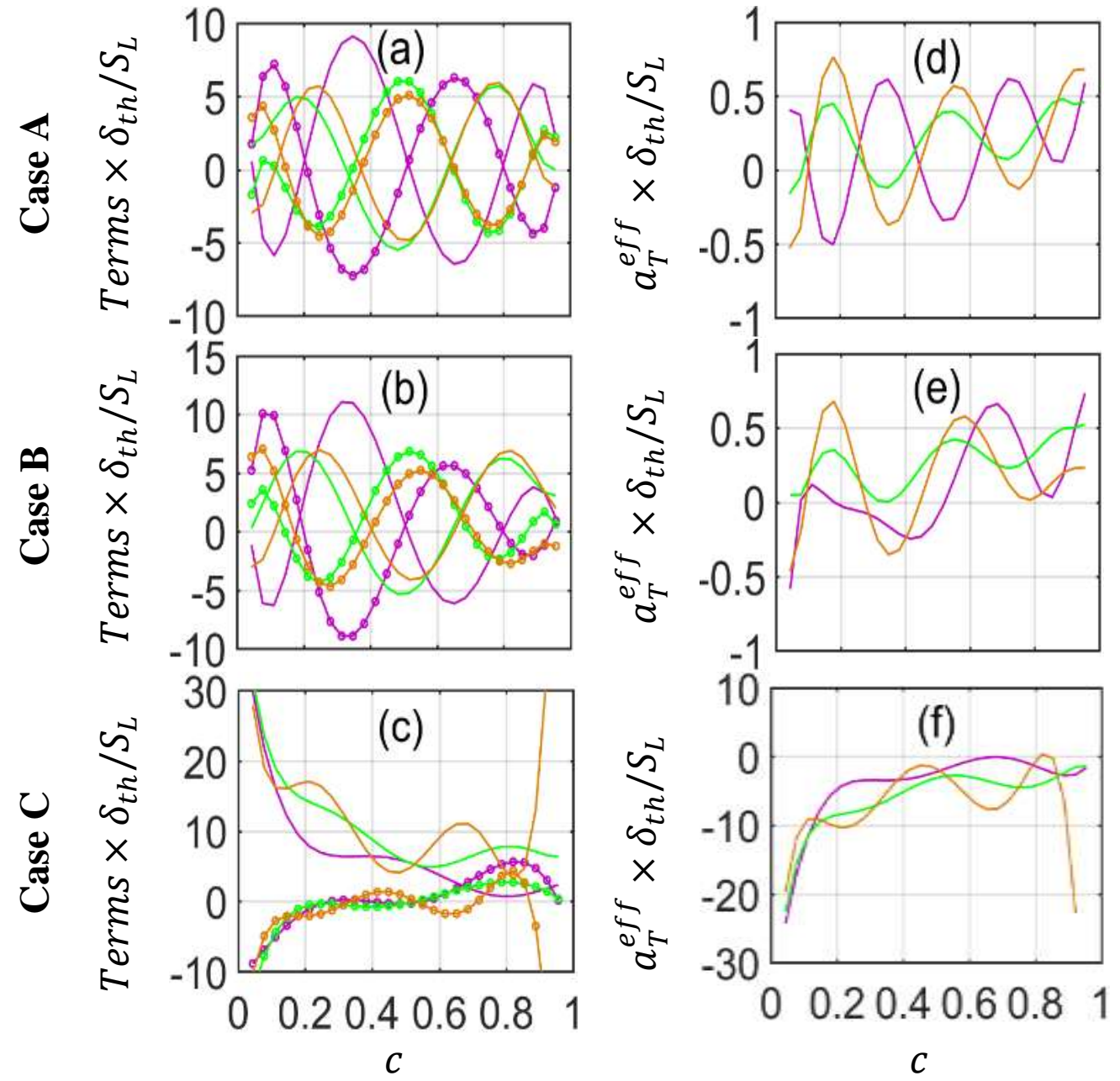

Fig. 7: Variations of (a-c) $a_{N}^{e f f}$, (solid line) $|\nabla c|^{-1}(\partial|\nabla c| / \partial t)$ (line with o) (1 ${ }^{\text {st }}$ column) and (d-f) $a_{T}^{e f f}\left(2^{\text {nd }}\right.$ column), normalized with $\delta_{t h} / S_{L}$, conditional upon $c$ for cases A-C ( $1^{\text {st }}-3^{\text {rd }}$ row). 